\title{
Wo ist der Speck, in den geschnitten werden kann?
}

\begin{abstract}
Milliardenzuwächse bei Umsatz und Ertrag, Spitzeneinkommen im Vergleich zu anderen Besserverdienenden - mit solchen Argumenten versucht der GKVSpitzenverband, die Koalition auf Einschnitte bei Ärzten zu konditionieren.
\end{abstract}

- Mit Blick auf die Haushaltsberatungen für 2011 und das voraussichtliche Defizit der Krankenkassen von elf Milliarden Euro ist der GKV-Spitzenverband mit einer Kampagne gegen die Arzthonorare an die Öffentlichkeit gegangen. Gezeigt werden soll, welche Teile der Gesellschaft in den letzten Jahren dicken Speck angesammelt haben, den anzugreifen keine Not auslöst.

Tatsache ist: Die GKV-Umsätze der Vertragsärzte und -Psychotherapeuten inklusive der Praxisgebühr sind zwischen 2007 und 2010 um 4,7 Milliarden Euro auf inzwischen 32,1 Milliarden Euro gestiegen. Das ist ein Zuwachs von $17,1 \%$ binnen drei Jahren nach einer durchschnittlichen Steigerungsrate von gut $2 \%$ in den zehn Jahren davor.

Die Große Koalition hatte den Ärzten einen gewissen Nachholbedarf zuge- standen und vor allem die Morbidität zum entscheidenden Kriterium für die Weiterentwicklung des Honorars gemacht. Das war ein Paradigmenwechsel, der die jetzige Regierung vor kaum lösbare Probleme stellt.
Neid auf die Ärzte wird geschürt
Den GKV-Spitzenverband kümmert das nicht. Er setzt auf den Neid der Öf- fentlichkeit und präsentiert dazu ein ganzes Bündel an Behauptungen, wie gut es Vertragsärzten im Vergleich zum Rest der Welt geht. So habe sich das durchschnittliche Gesamteinkommen (brutto) der Vertragsärzte von 126000 Euro im Jahr 2003 auf 164000 Euro in diesem Jahr verbessert. Datenbasis sind das Statistische Bundesamt und eine Hochrechnung auf das Jahr 2010, wo- bei für die letzten drei Jahre ein Kosten-

anstieg von $1 \%$ unterstellt wurde. Im Vergleich dazu verdient ein Oberarzt pro Jahr 99000 Euro. Diese Zahl gilt als betriebswirtschaftliche Kalkulationsgrundlage für das angemessene Arbeitsentgelt der Vertragsärzte. Die Folgerung ist klar: Die Arbeit des Vertragsarztes wird im Durchschnitt besser entgolten als die eines Oberarztes.

Erst recht verdienen Ärzte mehr als andere Besserverdienende, z.B. mehr als der Geschäftsführer (92 556 Euro), der Ingenieur (71 479 Euro) oder der Gymnasiallehrer (46 603 Euro). Deshalb hält die Kassenfront es für gerechtfertigt, ins Honorar der Ärzte zu schneiden: Minus 2,5\% ist die Forderung.

Dem steht das KBV-Petitum gegenüber, wachsende Morbidität mit 1,7\% und bisher unberücksichtigte Kostensteigerungen von rund zwei Milliarden Euro für das Honorar 2011 zu verhandeln. Nie war die Lücke zwischen Wunsch und Wirklichkeit so groß wie jetzt.

HELMUT LASCHET .

\section{AOK-Patienten können ihre Ärzte bewerten}

- AOK-Versicherte können seit Juni ihre Haus- und Fachärzte bewerten. Die AOKen und das Projekt Weisse Liste haben ihre neue Online-Arztsuche in den Pilotregionen Hamburg und Berlin gestartet, Thüringen soll in Kürze folgen. Erste Ergebnisse werden ab Herbst unter aok-arztnavi.de und weisse-liste.de veröffentlicht.

Die Bewertungen sollen Patienten wie Angehörigen helfen, den geeigneten Arzt zu finden. Der Fragebogen des Portals enthält Fragen zu den Bereichen Praxis und Personal, Arztkommunikation und Behandlung. Die Patienten sollen auch angeben, ob sie den Arzt an Freunde weiterempfehlen würden. Zielgerichtete Diffamierungen seien nicht möglich, betonen die Initiatoren. So seien Mindestmengen an Beurteilungen vorgesehen, um Ausgewogenheit zu garantieren. Auf Freitextfelder, die zu unsachlicher Kritik einladen, werde verzichtet. Beurteilte Ärzte hätten die Möglichkeit, die Beurteilungen für die Nutzer sichtbar zu kommentieren oder komplett sperren zu lassen.

Springer Medizin mit der „Ärzte Zeitung“" wird als Medienpartner ausführlich über das Projekt berichten. Informationen zu dem Projekt von AOK und Weisser Liste der BertelsmannStiftung einschließlich des Patientenfragebogens finden Sie unter www.aerztezeitung.de. 